\title{
Detection Approach for Botnets with Cross Cluster Correlation
}

\author{
V. Maruthi Prasad \\ Asst. Professor \\ Madanapalle Institute of \\ Technology \& Science \\ Madanapalle
}

\author{
K. Surekha \\ Asst. Professor \\ Madanapalle Institute of \\ Technology \& Science \\ Madanapalle
}

\author{
G. Naga Swetha \\ Research Scholar \\ Gurunankdev Engineering College \\ Bidhar
}

\begin{abstract}
Botnets are presently the key stage for some Internet assaults, for example, spam, dispersed foreswearing of-benefit (DDoS), fraud, and phishing. The vast majority of the current botnet identification approaches work just on particular botnet order and control (C\&C) conventions (e.g., IRC) and structures (e.g., brought together), and can progress toward becoming insufficient as botnets change their $\mathrm{C} \& \mathrm{C}$ strategies. In this paper, we present a general identification structure that is autonomous of botnet $\mathrm{C} \& \mathrm{C}$ convention and structure, what's more, requires no from the earlier information of botnets, (for example, caught bot parallels and henceforth the botnet marks, what's more, $\mathrm{C} \& \mathrm{C}$ server names/addresses). We begin from the definition and fundamental properties of botnets. We characterize a botnet as an organized gathering of malware occurrences that are controlled by means of $\mathrm{C} \& \mathrm{C}$ correspondence channels. The fundamental properties of a botnet are that the bots speak with some $\mathrm{C} \& \mathrm{C}$ servers/peers, perform malevolent exercises, and do as such in a comparative or related way. As needs be, our identification system groups comparative correspondence activity and comparative malevolent movement, and performs cross group connection to recognize the hosts that offer both comparative correspondence designs also, comparable vindictive movement designs. These hosts are in this way bots in the checked system. We have actualized our BotMiner model framework and assessed it utilizing numerous genuine system follows. The outcomes demonstrate that it can recognize certifiable botnets (IRC-based, HTTP-based, and P2P botnets including Nugache and Tempest worm), and has a low false positive rate.
\end{abstract}

\section{Keywords}

Command and Control Systems, Botnet, Botmaster

\section{INTRODUCTION}

The sharp increment of the web in the past period performed to have encouraged a development in the events of online attacks [1]. At the advanced time web is turning into the fundamental need of everybody. Today age is the time of distributed computing, which encourage the clients to access and store the information through cloud. Distributed computing is a portrayal for empowering all over, good, onrequest arrange access to an open, private and half and half shared pool of registering assets like stockpiling, administrations, server, systems, and application. These administrations can be furnished with least administration endeavours and rapidly. Gadgets which are associated with

the web are these days under the danger of various assaults performing through PC noxious programming's [2] [3]. The cloud servers can be gotten to through web, the more utilization of distributed computing drives the distributed computing toward the more digital assaults.

Botmaster control these tainted gadgets remotely through direction and control server. Botnet give the one-to-numerous relationship system among order and control server and bots, that is the reason the botmaster use botnet for ad, digital assaults, etc. When a gadget is tainted with pernicious code, it turns into the piece of a botnet, and begin working for the Botmaster without knowing to the end client. Botnet spread itself an opportunity to time by trading off an ever increasing number of gadgets as cell phones, PCs, PCs and diverse servers.

The quantities of digital assaults which are found in the web these days, most clients are influenced by these assaults are performed through botnet. Botmaster can perform distinctive sort of cybercrime like DDoS, click misrepresentation, phishing extortion, key logging, bit coins' extortion, spamming, sniffing traffic, spreading new malware, google AdSense maltreatment with bots [6]. These days the botnet is turning into the base of all cybercrime which is performed through the internet [7][8]. Botmaster utilize distinctive strategies to contaminate a client gadget to make it bot (zombie) like drive by download, email and pilfered programming's are the most well-known method for attacks [9][10]. As per the past research bunches of the discovery approaches have been proposed. Be that as it may, the greater part of them are centered around the disconnected location of botnet; still we have to concentrate on the ongoing detection [11]. The current botnet identification procedures are sort into two principle bunches given as Honeynets Based Detection Technique and Intrusion Detection System [12]. Scientists center around the digital security to recognize botnets assaults and keep cloud servers from the botnet assaults. Yet at the same time inquire about on botnet location is juvenile, and need more research to enhance information security in distributed computing.

\section{PROBLEM DEFINITION}

As indicated by the definition given over, a botnet is described by both a C\&C correspondence channel (from which the botmaster's directions are gotten) and malignant exercises (when directions are executed). Some different types of malware (e.g., worms) may perform malevolent exercises, however they don't associate with a C\&C channel. Then again, some typical applications (e.g., IRC customers and ordinary $\mathrm{P} 2 \mathrm{P}$ record sharing programming) may demonstrate correspondence designs like a botnet's $\mathrm{C} \& \mathrm{C}$ channel, however they don't perform malevolent exercises. Figure 1 shows two commonplace botnet structures, in particular incorporated and $\mathrm{P} 2 \mathrm{P}$. The bots get directions from the botmaster utilizing a push or draw instrument and execute the allocated 
undertakings.

The activity of an incorporated botnet is moderately simple and instinctive, though this isn't really valid for P2P botnets. Thusly, here we quickly show a case of a run of the mill P2Pbased botnet, specifically Storm worm. So as to issue directions to the bots, the botmaster distributes/shares order records over the $\mathrm{P} 2 \mathrm{P}$ organize, alongside explicit pursuit keys that can be utilized by the bots to locate the distributed order documents. Tempest bots use a force system to get the directions. In particular, every bot much of the time contacts its neighbor peers hunting down explicit keys so as to find the related direction documents. Notwithstanding seek tasks, the bots likewise often speak with their companions and send keep-alive messages. In both incorporated and P2P structures, bots inside the equivalent botnet are probably going to carry on also regarding correspondence designs. This is generally because of the way that bots are non-human driven, precustomized to play out a similar routine $\mathrm{C} \& \mathrm{C}$ rationale/correspondence as composed by the equivalent botmaster.

In the brought together structure, regardless of whether the location of the $\mathrm{C} \& \mathrm{C}$ server may change regularly (e.g., by oftentimes changing the A record of a Dynamic DNS area name), the $\mathrm{C} \& \mathrm{C}$ correspondence designs stay unaltered. On account of P2P-based botnets, the friend correspondences (e.g., to look for directions or to send keep-alive messages) pursue a comparable example for every one of the bots in the botnet, albeit every bot may have an alternate arrangement of neighbor peers and may impact on various ports. Despite the particular structure of the botnet (brought together or P2P), individuals from the equivalent botnet (i.e., the bots) are composed through the $\mathrm{C} \& \mathrm{C}$ channel. When all is said in done, a botnet is not quite the same as a lot of separated individual malware examples, in which each extraordinary occurrence is utilized for an entirely unexpected reason.

In spite of the fact that in an outrageous case a botnet can be arranged to deteriorate into a gathering of segregated hosts, this isn't the normal case. In this paper, we center around the most run of the mill and helpful circumstance in which bots in the equivalent botnet perform comparable/facilitated exercises. To the best of our insight, this remains constant for the majority of the current botnets saw in nature. Tocondense, we accept that bots inside the equivalent botnet will be described by comparable vindictive exercises, just as comparable $\mathrm{C} \& \mathrm{C}$ correspondence designs. Our suspicion holds even for the situation when the Botmaster separates a botnet into sub-botnets, for instance by doling out various undertakings to various arrangements of bots.
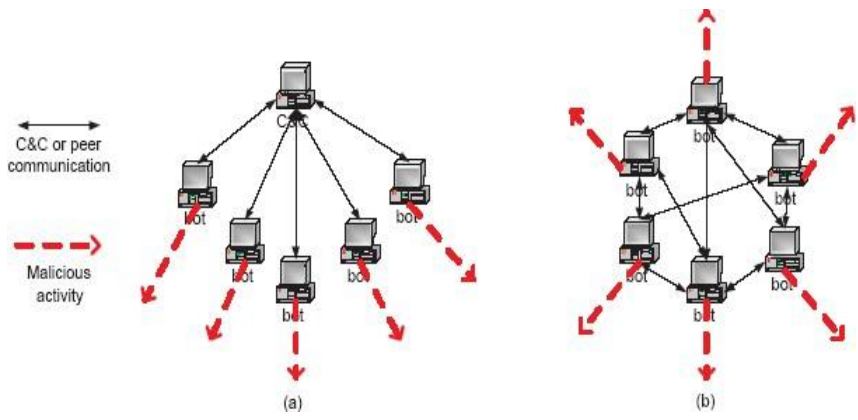

Figure 1: Centralized and Peer to Peer BOTNET

\section{ARCHITECTURE OF BOTMINER}

\subsection{Methodology}

More specifically, our detection framework clusters similar communication activities in the C-plane (C\&C communication traffic), clusters similar malicious activities in the A-plane (activity traffic), and performs cross cluster correlation to identify the hosts that share both similar communication patterns and similar malicious activity patterns. These hosts, according to the botnet definition and properties discussed above, are bots in the monitored network. This system makes the following main contributions.

- We develop a novel general botnet detection framework that is grounded on the definition and essential properties of botnets. Our detection framework is thus independent of botnet $\mathrm{C} \& \mathrm{C}$ protocol and structure, and requires no $a$ priori knowledge (e.g., C\&C addresses/signatures) of specific botnets. It can detect both centralized (e.g., IRC, HTTP) and current (and possibly future) P2P based botnets.

- We define a new "aggregated communication flow" (Cflow) record data structure to store aggregated traffic statistics, and design a new layered clustering scheme with a set of traffic features measured on the C-flow records. Our clustering scheme can accurately and efficiently group similar $\mathrm{C} \& \mathrm{C}$ traffic patterns.

- We build a BotMiner prototype system based on our general detection framework, and evaluate it with multiple real-world network traces including normal traffic and several real-world botnet traces that contain IRC, HTTP and P2P-based botnet traffic (including Nugache and Storm). The results show that BotMiner has a high detection rate and a low.

The below chart comprises of predominantly five segments.

C-plane and A-plane, C - Plane and A - Plane grouping, Cross -Plane Correlation 


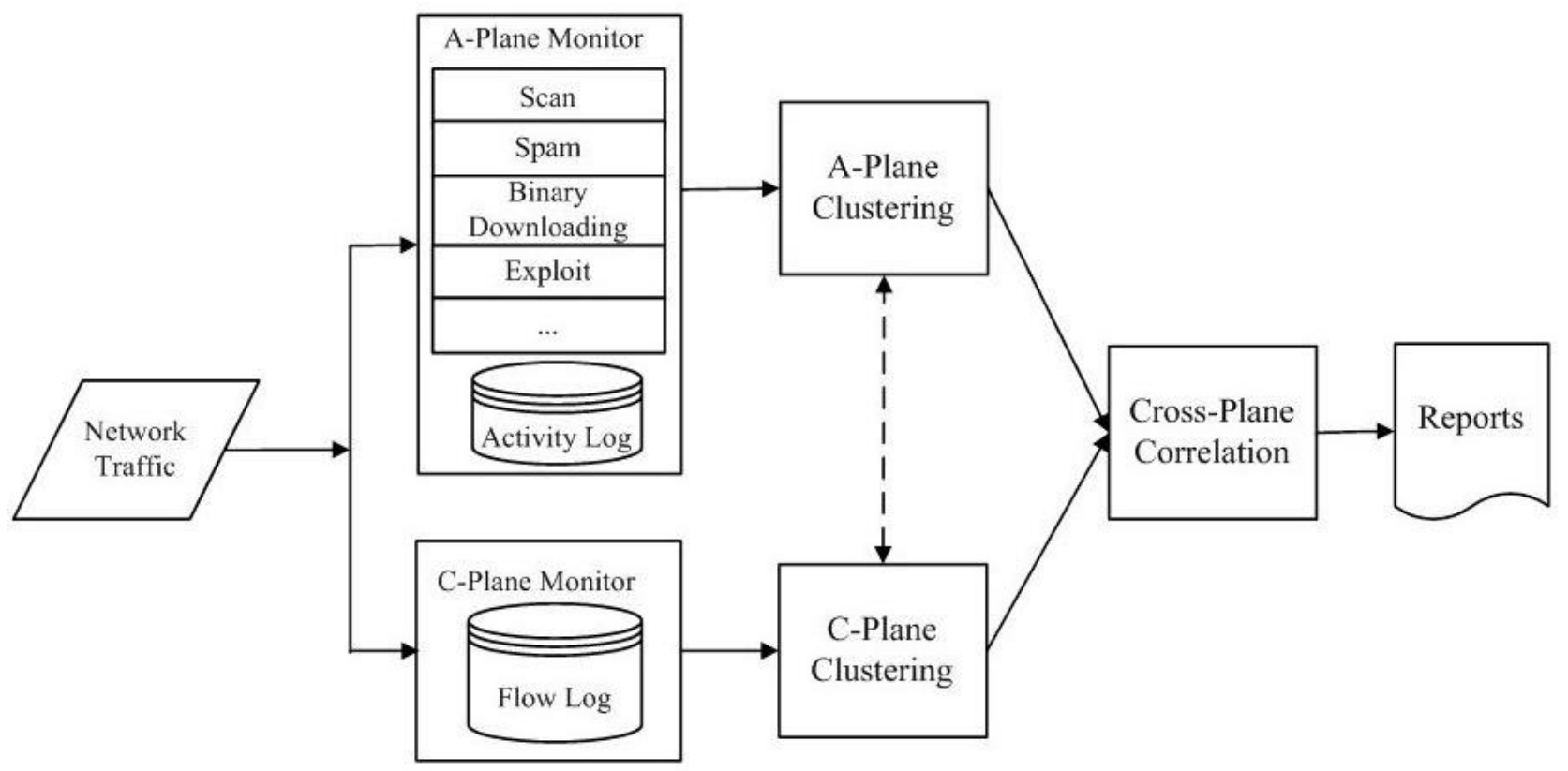

Figure 2: Architecture of Botminer Detection Framework

\section{C-plane and A-plane}

The two traffic screens in C-plane and A-planecan be sent at the edge of the system analyzing traffic among inside and outside systems, like BotHunter and BotSniffer. They keep running in parallel and screen the system traffic. The $\mathrm{C}$-plane screen is in charge of logging system streams in a configuration reasonable for effective capacity and further examination, and the A-plane screen is in charge of identifying suspicious exercises (e.g., checking, spamming, and misuse endeavours).

\section{$\mathrm{C}$ - Plane and $\mathrm{A}$ - Plane grouping}

The C-plane grouping and A-plane bunching segments process the logs created by the C-plane and A-plane screens, individually. The two modules remove various highlights from the crude logs and apply bunching calculations so as to discover gatherings of machines that indicate fundamentally the same as correspondence (in the C-plane) and action (in the A-plane) designs.

\section{Cross - Plane Correlation}

It joins the aftereffects of the C-plane and A-plane grouping and settles on an official conclusion on which machines are potentially individuals from a botnet. In a perfect circumstance, the traffic screens ought to be disseminated on the Internet, and the screen logs are accounted for to a focal storehouse for grouping and cross-plane investigation.

For advancement of these Botminer we are executing three distinct sorts related modules.

These are as per the following

\section{BotNet}

\section{Victim 3. BotMiner}

\section{Existing System:}

Some ordinary applications (e.g.,IRC customers and typical $\mathrm{P} 2 \mathrm{P}$ record sharing programming) may indicate correspondence designs like a botnet's C\&C channel, however they don't perform vindictive exercises. We center around the most average and helpful circumstance in which bots in the equivalent botnet perform distinctive action. Our A-plane screen is manufactured dependent on Snort, an open- source interruption recognition apparatus. BotMiner is a novel general identification framework that does not have such impediments and can significantly supplement existing discovery approaches.

\section{Proposed System:}

A botnet is portrayed by both a $\mathrm{C} \& \mathrm{C}$ correspondence channel (from which the botmaster's directions are gotten) and malevolent exercises (when directions are executed). Some different types of malware (e.g., worms) may perform malignant exercises, however they don't interface with a $\mathrm{C} \& \mathrm{C}$ channel. We adjusted existing interruption recognition methods and executed them as Snort pre-processor modules or marks. BotMiner is a novel general location framework have an impediments and can significantly supplement discovery approaches.

\section{ALGORITHMS}

\subsection{Botnet}

Step 1. First connect to Botminer

Step 2. Select net IP and change IP address

Step 3. Load and process data Send the file

Step 4. File received.

Step 5. Connect to victim

Step 6. Load and Process victim

Step 7. Check victim file is connected or not.

\subsection{Victim}

Step 1. Loading and process of Victim file

Step 2. Select IP addresses

Step 3. Check botnet is activated or nor

Step 4. Activated protect the file form Botminer

Step 5. Process and load the file 


\subsection{Botminer Main}

Step 1. Connect to BootMaster folder

Step 2. Select net IP and change IP address

Step 3. BotNet folder another system (client)

Step 4. Victim folder another system(server)

Step 5. Load and process both BootNet and victim

Step 6. Choose file to be send

Step 7. Give victim system IP address

Step 8. Activate and protect the file from boot master

Step 9. BotNet receive the content and forward to the victim system.

\section{CONCLUSION}

Botnet discovery is a testing issue. In this paper, we proposed a novel system inconsistency based botnet location framework that is free of the convention and structure utilized by botnets. Our framework abuses the fundamental definition and properties of botnets, i.e., bots inside the equivalent botnetwill display comparable C\&C correspondence designs and comparable vindictive exercises designs. In our trial assessment on some true system follows, BotMiner indicates superb discovery precision on different kinds of botnets (counting IRC-based, HTTP based, and P2Pbased botnets) with a low false positive rate on ordinary traffic. Almost certainly, future botnets (particularly P2P botnets) may use avoidance procedures to dodge discovery, as talked about in Section 4. In our future work, we will contemplate new methods to screen/bunch correspondence and action examples of botnets, and these strategies are expected to be progressively strong to avoidance endeavours. What's more, we intend to additionally enhance the productivity of the C-stream changing over and bunching calculations, consolidate diverse connection strategies (e.g., vertical relationship and flat relationship), and grow new continuous location frameworks dependent on a layered structure utilizing examining methods to work in rapid and extensive system situations.

\section{REFERENCES}

[1] E. Alomari, "Botnet-based Distributed Denial of Service ( DDoS ) Attacks on Web Servers: Classification and Art," vol. 49, no. 7, pp. 24-32, 2012.

[2] M. Thapliyal, N. Garg, and A. Bijalwan, "Botnet Forensics : Survey and Research Challenges," no. April, 2013.

[3] F. Carpine and S. Maria, "Online IRC Botnet Detection using a SOINN Classifier," pp. 1351-1356, 2013.

[4] R. A. Rodr, I. Omez, G. M. A-fern, and P. Garc, "Survey and Taxonomy of Botnet Research through Life-Cycle," vol. 45 , no. $4,2013$.

[5] I. Ullah, N. Khan, and H. a. Aboalsamh, "Survey on botnet: Its architecture, detection, prevention and mitigation," 2013 10th IEEE Int. Conf. NETWORKING, Sens. Control, pp. 660-665, Apr. 2013

[6] S. S. C. Silva, R. M. P. Silva, R. C. G. Pinto, and R. M. Salles, "Botnets: A survey," Comput. Networks, vol. 57, no. 2, pp. 378-403, Feb. 2013.

[7] "Botnets The New Threat Landscape White Paper [Threat Control] - Cisco Systems.".

[8] M. Zahid, A. Belmekki, and A. Mezrioui, "A new architecture for detecting DDoS/brute forcing attack and destroying the botnet behind," 2012 Int. Conf. Multimed. Comput. Syst., pp. 899-903, May 2012.

[9] W. Paper, "Anatomy of a Botnet." [10] "Microsoft Security Intelligence Report," vol. 15, 2013. [11] W. Xianghua and C. Lijun, "Analysis and Design of Botnet Detection System," 2012 Int. Conf. Comput. Sci. Serv. Syst., pp. 947-950, Aug. 2012 